\title{
Practical Considerations of Convection Enhanced Delivery for Novel Therapies Treating Spinal Cord Injury Related Neuropathic Pain
}

\author{
Gurwattan S Miranpuri ${ }^{1}$, PhD, Karl Sillay ${ }^{2}$, MD, Wendell B. Lake ${ }^{1}$, MD MS, \\ Daniel K. Resnick ${ }^{1^{*}}$, MD, MS \\ ${ }^{I}$ Department of Neurological Surgery, University of Wisconsin School of Medicine and Public Health, Madison, \\ WI 53792, USA \\ ${ }^{2}$ Department of Anatomy and Neurobiology, University of Tennessee Health Sciences Center, USA
}

*Corresponding Author: Daniel K. Resnick, Department of Neurological Surgery, University of Wisconsin School of Medicine and Public Health, Madison, WI 53792,USA, Email: resnick@neurosurgery.wisc.edu

\begin{abstract}
The incidence of traumatic spinal cord injury (SCI) in the United States is around 12,500 new cases per year with a total prevalence of roughly 276,000 patients. Neuropathic pain (NP) is present in $70 \%$ of patients suffering from SCI and is a significant concern.

Most research focuses on small animal models with small spinal cords (SC) where simple diffusion of a pharmaceutical or biologic treatment can have a substantial effect. However, promising molecular, gene and cell therapies for treating SCI and NP often fail to demonstrate efficacy in the much larger human SC where simple diffusion is not strong enough to bring the therapy into a significant portion of the transverse spinal cord.

Additionally, there is a need to visualize, target and control delivery in humans to specific gray matter SC tracts to generate the desired treatment while avoiding undesired side effects. The development of these promising therapies, however, significantly hampered by the lack of a platform for targeted delivery of therapeutics and lack of a relevant large animal model. To address hurdles in delivering agents across the blood/SC barrier, we propose to utilize a CED injection technique that delivers therapeutic agents into neural tissue using a positive pressure gradient that expands the interstitial space and increases fluid penetrance; we have successfully employed CED to overcome limitations of drug delivery in the brain.

From clinical perspective, evidence makes it clear that miRNAs may manipulate cell state and molecular processes in order to enhance functional recovery following SCI and alleviate NP. This review contains the extensive history of the use of modeling CED in the spinal cord and understanding the role of siRNA, miRNA, TIMP therapies and limitations if any. This knowledge can serve as a foundation in the development of minimally invasive CED platforms for the delivery of therapeutics into targeted regions of the SC for the attenuation and repair of SCI induced NP.
\end{abstract}

Keywords: Spinal Cord injury, SCI, neuropathic pain, NP, matrix metalloproteinase, MMP, tissue inhibitor of MMP, TIMP, miRNAs, siRNAs, convection enhanced delivery, CED, MRI

\section{INTRODUCTION:}

Processes such as trauma, neurodegenerative disorders, inflammation, and infection may result in spinal cord injury (SCI) and associated neuropathic pain (NP). NP can be difficult to treat and presents an opportunity for advancing research on several issues related to pain, explicitly relating to transcriptional, epigenetic and post-transcriptional research [1]. Effective treatment for relief of NPis currently limited; however, direct delivery of either cellular or biologically active therapeutics into the spinal cord may offer an opportunity for treatment of NP. Distinct role of matrix metalloproteinase MMP9 and MMP2 reported recently in causing NP following spinal nerve ligation in a rodent model [2]. MMP-9 induces NP through interleukin- $1 \beta$ cleavage and microglia activation at early phase of injury and MMP2 maintains NP through interleukin-1 $\beta$ cleavage and astrocyte activation at later phase of injury. Intrathecal injections of tissue inhibitors TIMP- 
1 and TIMP-2 resulted in significant suppression of NP [2].The study suggested that a dual inhibition of MMP-9 in early phase and MMP2 in late phase of injury alleviate NP. Using Sprague Dawley rat, we have established a contusion SCI-induced NP model that demonstrates expression and activity of MMP9 in early phase and MMP2 in late phase of injury. These results are supported by molecular evidence of $\beta$-catenin involved in the WNT signaling pathway, and extracellular signaling kinase within the MAPK cascade (Miranpuri et al., 2016) [3]. Given the limitations of diffusion, larger animals with correspondingly larger spinal cords may require direct infusion of the therapeutics into the spinal cord parenchyma to realize results. However, there is currently no reliable method for infusion of therapeutic agents directly into the spinal cord. In a review published in 2010 from our lab summarized the findings related to the role of RNAi in vivo to study chronic pain and limitations involved in therapeutic agents' delivery methods. The challenges involve in used methods of delivery (intravenous or oral) of siRNA to the CNS in sufficient concentrations because siRNA does not cross the blood brain barrier (BBB) and gets degrade in the blood by end nucleases. Furthermore, delivery could be improved using transfection agents as (cationic lipid i-Fect ${ }^{\mathrm{TM}}$, polyethyleneimine, and chitosan-nanoparticles) enabling siRNA directly to the spinal cord through intrathecal injection but the effects of direct delivery have been relatively short-lived.

Newly emerging convection enhanced delivery (CED) technique offers an alternate method to deliver a high concentration of biological therapeutic agents that otherwise do not cross the blood-brain barrier [5].Despite the promise of CED, several technical barriers such as those relating to the infusate trajectory reflux and poor drug distribution have limited its effectiveness [6]. Drug therapy using direct CED in brain been reported for treating glioma [7] and epilepsy [8]. CED-based in vitro, ex vivo and in vivo animal models have led to active genetherapy trials for treating Parkinson disease (PD)and are supported by histological studies on disease pathology [9].Use of MRI navigation in some clinical trials, helps accurately targeting and providing real time monitoring of viral vector delivery (rCED). Several researcher labs including ours adopted different small and large animal models exploring use of CED in brain [9-26]. The results from these studies are promising with a suggestion of superiority over intrathecal (IT) injection. Most of these studies have demonstrated that neuronal growth factorinduced enhancement of endogenous neurorestoration may improve neuronal connectivity after severe neurologic injury, particularly if delivered intraparenchymally with zero-order kinetics [26]. The aforementioned experiments suggest that CED technologies will likely have a role in treating SCI, NP, gliomas and other CNS diseases in the future.

The role of $\sim 20-30$ nucleotide RNA molecules known as short interfering (siRNAs) and miRNAs have been reported as critical regulators in the expression and functionality of eukaryotic genomes [27]. Their implication in gene expression and control are generally inhibitory, making the corresponding regulatory mechanism fall under RNA silencing. There are current limitations in the use of RNAi short response as a delivery technique to stimulate RNAi through the cell's natural mechanisms. A mini review from our lab documented the work done on pain using RNAi in vivo [4].

Recently, several studies have demonstrated the possible role of miRNAs as alternative therapeutic approach for treating SCI-induced NP. In animal models, the dorsal root ganglia, the spinal dorsal horn, and the brain are all areas of miRNA modulation [28]. Following injury, the differential expression of miRNAs in the nervous system may play a role in predicting the development of chronic pain [29]. CED has not been explored extensively as a delivery method for miRNAs in the treatment of NP, recent studies have identified several microRNAs including miR-21, miR-486, miR-20 involved in key mechanisms of SCI such as cell death or astrogliosis, direct evidence on the role of miRNAs in SCI is scarce [30]. Furthermore, from clinical perspective, evidence makes it clear that miRNAs may be potent therapeutic tools that can manipulate cell state and molecular processes in order to enhance functional recovery. In this paper, we will review the extensive history of the use of modeling CED in the brain and spinal cord and discuss the role of siRNA and miRNA therapies in alleviating NP (Figure 1) 


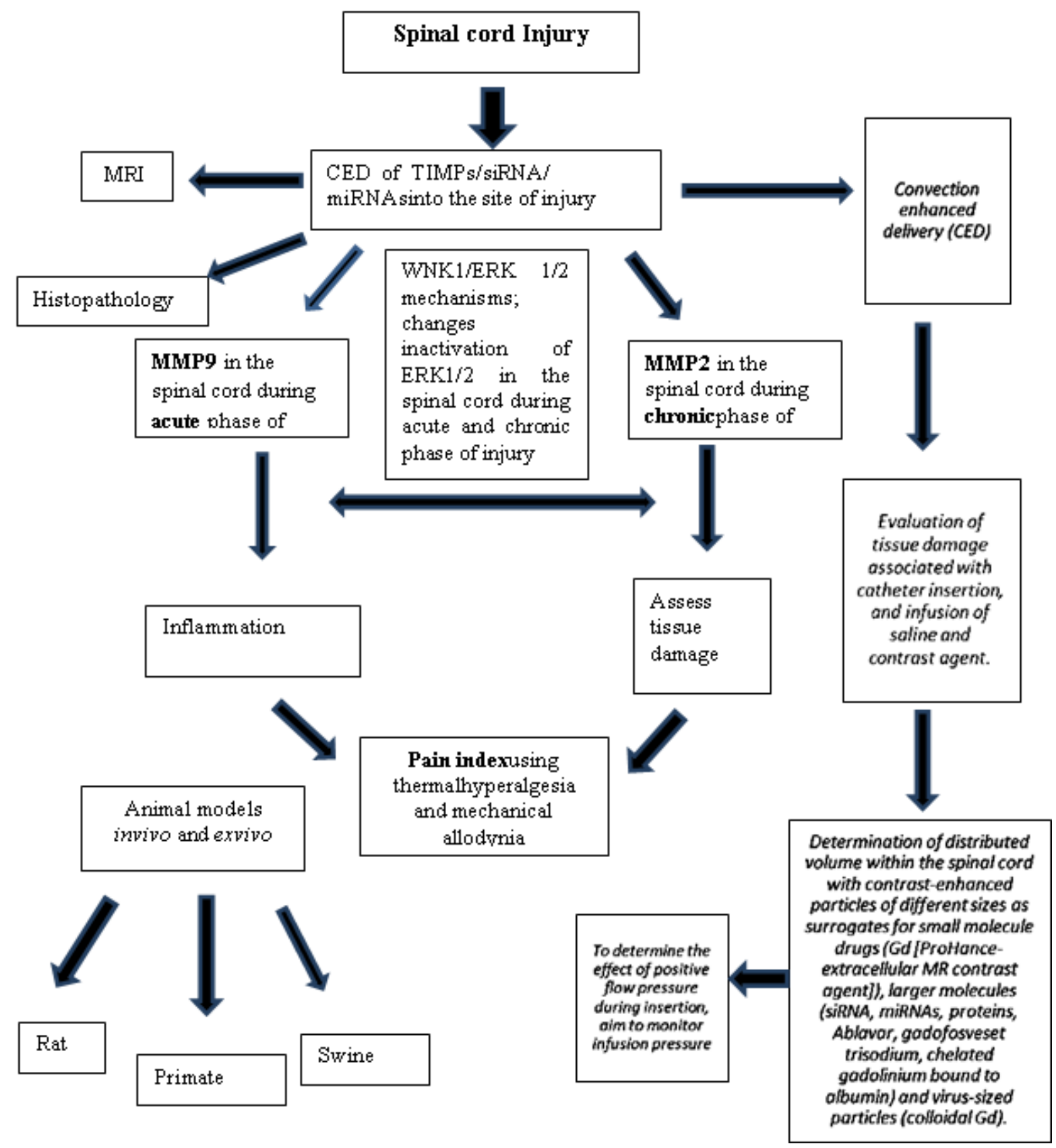

Figure1. Practical approach of convection enhanced delivery of therapeutic agents in spinal cord

\section{Modeling CED In THE BRAin}

\subsection{Delivery System and Target Tissue Testing}

CED in the brainstem tested as a safe alternative method for treating diffuse pontine gliomas in rats showing minimal neurological deficit and tissue disruption [31]. CED technique was introduced in 2003 became popular as techniques have shifted the bench to the clinic [32].Hundreds of references appeared regarding modeling CED in brain. However, very little information is available on CED specifically in the spinal cord. In this review, we shift our discussion of CED in the brain to focus on knowledge concerning CED in the spinal cord. Modeling CED therapy delivery via in vitro, ex vivo and in vivo animal models and translation to the human for treating PD has allowed insight into technical factors associated with CED [10]. Apparatus and technique fundamentals are testable in gel infusion models employing CED to facilitate macromolecules that could not penetrate the blood brain barrier. Example of technical details affecting infusion success includes validating consistency and reliability of the surrogate tissue infusion $[33,34]$. 
Once the surrogate tissue model is felt stable, factors such as, CED infusion catheter systems including backflow, infusion cloud morphology, volume of distribution versus the infused volume, rate of infusion and pressure to ensure performance of candidate catheter designs including smart catheters using micro-electromechanical-systems (MEMS) [5, 6].Similarly, infusion pressure, flow rate, and volume changes affect infusion success be evaluated across catheter designs [12]. Methods of predicting and detecting infusion protocol variance such as monitoring infusion line pressure changes and altering the infusion to avoid aberrant infusion morphology and catheter reflux are helpful in maximizing therapy are testable in the surrogate brain model. Furthermore, the strategies for the movement of an infusion catheter within the target tissue with respect to order of infusion versus movement and catheter design are testable [5]. Commercially available systems for low volume, pressure monitored infusions may also be tested within the surrogate brain to determine infusion pump requirements for planned and future infusion protocols, including operating requirements such as MRI safety of the infusion system [11]. Findings during research on CED catheter design and development of CED stroke treatment lead to the merger of ultra-sound and CED.CED in regard to volume distributed (Vd), volume infused ( $\mathrm{Vi}$ ) and location using in vitro model, ex vivo brain animal models, in vivo insect, mouse, rat, and non-human primate models of PD have been reported in an effort to validate techniques for benchmarking infusion characteristics across models $[9,10]$ and adopted for use in human gene-based therapy trials [10].

\subsection{Targeted therapeutic testing}

Once the targeted therapy delivery factors including location, concentration gradient delivered, and complications such as incomplete target coverage and catheter reflux demonstrated, the modeling comparisons become helpful regarding the specific targeted therapeutic agent. Targeted therapeutics tested in the brain model include viral vector therapies $[20,35,36,37]$.

\section{Modeling CED In The SPINAl CORD}

Penetration of macromolecules across the bloodspinal cord barrier is limited for several therapeutic compounds that have potential for treating spinal cord disorders. Macromolecules delivered, distributed regionally in a predictable and homogeneous manner and over significant volumes of naive and traumatized rat spinal cord by employing CED [39]. These characteristics make CED a valuable tool to investigate the therapeutic potential of various compounds for the treatment of injury and spinal cord diseases [39].Regional convective delivery provides reproducible, safe, region-specific, and homogeneous distribution of macromolecules over large longitudinal segments of the spinal cord. This delivery method overcomes the various obstacles associated with current delivery techniques, as suggested by the increase in the mean $\mathrm{Vd} / \mathrm{Vi}$ ratio in corresponding studies of macromolecule administration via CED [39, 40, 41]. These findings call for further research done in order to investigate new treatments of various conditions of the spinal cord.

The correlation of injected vector, AAVthymidine kinase (AAV-TK) with varying doses and its distribution into rat brain via CED revealed a clear dose response[42].CED of AAV-thymidine kinase into the rat brain showed that both subcutaneous osmotic pumps and infusion pumps were able to deliver vectors. However, osmotic pumps resulted in toxicity around brain tissue. Three weeks following $\mathrm{CED}$, vectors were present in both hemispheres of the brain, spinal cord, spleen, and kidney. However, the viability of delivering Adenoassociated virus (AAV-) based vectors to the spinal cord with CED in a rat model not been tested.

Convective delivery to lesion sciatic nerves tested in a rat and primate model with similar injuries [43]. Infusions encounter failure in the first 10 days after injury, the infusion fails to cross the site of injury in crushed or lacerated nerves. Following 14 days of crush injury CED is able to perfuse through the site of injury into the nerves of rats. In primates, the radioactively labeled albumin infuses into the nerve by day 28 . The study shows that in laceration injuries, successful convection delivery only occurs when nerve genesis is complete. CED does allow for successful delivery of macromolecules to the site of injury. Could the injured spinal cord also have limited diffusion via CED similar to nerves, needs to confirm. 
Table1. Three Key research findings of CED in the spinal cord of primate, rat and swine model

\begin{tabular}{|c|c|c|}
\hline Inquiry & Experimental Design & Findings \\
\hline 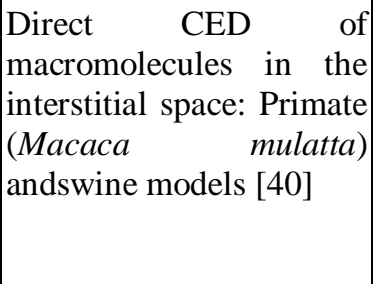 & $\begin{array}{l}\text { Silica cannula: (ID } 100 \mathrm{~mm} \text {, OD } 170 \mathrm{~mm} \text { ); } \\
\text { Albumin: unlabeled or labeled with carbon-14 } \\
\text { or gadolinium }(3,6,10,20,40 \text {, or } 50 @ 0.1 \\
\text { ml/min into the dorsal columns of swine and } \\
\text { into the lateral columns of primates }\end{array}$ & $\begin{array}{l}\text { Macro molecule perfusion resulted in } \\
\text { mean } \mathrm{Vd} / \mathrm{Vi}=4.4+/-0.5 \mathrm{ratio} \text {; } \mathrm{Vd} \text { got } \\
\text { escalated with increase in } \mathrm{Vi} \text { that } \\
\text { allowed large segments of spinal cord } \\
\text { (up to } 4.3 \mathrm{~cm} \text {; Vi } 50 \mathrm{ml} \text { ); Infused } \\
\text { albumin from the spinal cord } 85.5+/- \\
5.6 \% \text {. }\end{array}$ \\
\hline \begin{tabular}{|lrr|} 
Direct & convective \\
delivery & & of \\
macromolecules & to \\
peripheral & nerves: & A \\
primate & \multicolumn{3}{c}{$($ Macaca } \\
mulatta) & model [41]
\end{tabular} & $\begin{array}{l}\text { Small silica cannula inserted in peripheral } \\
\text { nerve; }(10,20,30,40 \text {, and } 80 \mathrm{ml}) \text { of } 14 \mathrm{C}- \\
\text { labeled or gadolinium-labeled albumin were } \\
\text { infused unilaterally or bilaterally into the tibial } \\
\text { nerves of primates @ } 0.5 \mathrm{ml} / \mathrm{min} \text {; and one } \\
\text { primate infused bilaterally with gadolinium- } \\
\text { bound albumin ( } 40 \mathrm{ml} / \text { nerve) underwent } \mathrm{MR} \\
\text { imaging } 16 \text { weeks after infusion. }\end{array}$ & $\begin{array}{l}\text { Mean Vd/Vi ratio was } 3.7+/-0.8 \\
\text { filled long segments of nerve (up to } \\
6.8 \mathrm{~cm} \text { ); radioactivity recovery } \\
75.8+/-9 \% \text {; no neurological deficits. }\end{array}$ \\
\hline $\begin{array}{lr}\text { Convective delivery of } \\
\text { macromolecules rinto } \\
\text { non-traumatized r } \\
\text { traumatized spinal cords: } \\
\text { A rat model [39] }\end{array}$ & $\begin{array}{l}\text { Infusion of } 1,2 \text {, and } 4 \mathrm{ml} \text { of } 14 \mathrm{C} \text {-albumin } \\
\text { infused into the dorsal columns of naive and } \\
\text { traumatized rat spinal cords. }\end{array}$ & $\begin{array}{l}\text { Non-traumatized group, } \mathrm{Vd} / \mathrm{Vi} \text {, } \\
4.3+/-0.6 ; \text { the traumatized spinal } \\
\text { cords, the } \mathrm{Vd} / \mathrm{Vi} \text { ratio }(3.7+/-0.5) ;\end{array}$ \\
\hline
\end{tabular}

The use of intraparenchymal infusions in the treatment of localized chronic pain supported by data analyzing the distribution of substance $P$ (SP) nocitoxins in to the human dorsal column [44]. A constant infusion of SP-diphtheria toxin fusion protein (SP-DT'@0.1 $\mathrm{ml} / \mathrm{min}$ ) exhibited a transverse directed distribution of cytotoxicity in NK1 receptor-expressing neurons within six to eight hours. Catheter placement, high infuscate concentration, and a stable SP analog all favor efficient treatment and distribution. To predict spatial drug distribution in nervous tissue, 3D models needed when analyzing drug delivery methods such as CED [45]. Diffusion tensor imaging (DTI) accurately predicts small volumes of CED into the dorsal white matter column. However, with larger infusion volumes (>2 $\mathrm{ml}$ ), albumin loss is greater than predicted.

Single-dose peripheral (intraperitoneal) treatment was compared to CED with relation to use of the anti-West Nile virus neutralizing humanized monoclonal antibody, hE16, in the treatment of WNV infected hamsters [46]. CED via direct administration into the pons protected the hamsters from lethal infection for greater time compared to peripheral treatment. Intraperitoneal administered $\mathrm{hE} 16$ identified in a time-dependent manner in the serum, CSR, cerebral cortex, brain stem and spinal cord. A complex fiber-tract organization necessary to enhance the accuracy of diffusion tensor imaging (DTI) -based methods. Another study utilized a 3D interstitial transport modeling approach in which tissue properties and anatomical boundaries assigned on a voxel-byvoxel basis using tissue alignment data from DTI [47]. The model estimates interstitial transport in isotropic and anisotropic tissues with the porous media transport theory. Distribution volumes were comparable for small infusion volumes $(<4 \mathrm{ml})$. DTI methodology allows interstitial flows and tracer distributions estimated more quickly following CED into the spinal cord. Quantifying the transport fields is important in order to develop drug-specific models for infusion.

Post-infusion spatial distribution of therapeutic agents is a vital measurement in determining the effectiveness of disease treatment and for perfecting direct tissue infusion technique [48]. Gd-labeled tracers (Gd-albumin), enhanced via MRI, are used to indicate concentration distribution. Gd-tracer technique used for measuring direct tissue infusion effectiveness, when treating nervous system diseases. CED into peripheral nerves might be useful for delivering therapeutics to the intrathecal space or to the fiber bundles entering the spinal cord [49]. The extracellular transport dynamics of Gd-DTPA-albumin tracer during CED into rat sciatic nerves was described in vivo using highresolution dynamic contrast-enhanced MR imaging at 11.1 Tesla. Tracers followed 
peripheral nerves towards the spinal cord and entered the cerebrospinal fluid and nerve roots at levels L4 and L5. Direct uptake into spinal cord tissues was limited. CED factors such as cannula placement, and underlying tissue structures affected spatial distribution patterns within spinal cord regions. CED into peripheral nerves is able to deliver therapeutics to nerve roots and to intrathecal space surrounding the spinal cord, making it a viable therapeutic tool [49].

While investigating brain fatal diseases like GM2 gangliosidoses, intracranial co-injection into month-old Sandhoff mice of recombinant adeno-associated viral vectors (rAAV) expressing human $\beta$-hexosaminidase $\alpha$ (HEXA) and $\beta$ (HEXB) prevented disease throughout the brain and spinal cord [50]. Gene transfer confined to the striatum or cerebellum prevented typical symptoms of disease. Infusion of rAAV into CSF space and intraparenchymal administration by CED is able to treat neurodegeneration in several disease states that affect nervous system functioning. BDNF delivered via a micro cannula attached to a sixweek mini-osmotic pump inserted into the spinal cord parenchyma between the site of nerve insertion and hemi section model of rat improved the connectivity at the peripheral motor bridge [51]resulting in hind leg electromyogram response of shorter latency and larger amplitudes, suggesting that therapeutic delivery via CED is effective.

\section{TIMPS THERAPY}

MMP-9 induces NP, implicated in IL-1 $\beta$ microglia activation in the DRG and spinal cord. Recombinant TIMP-1, tissue inhibitor of MMP9 , injections $(4.5 \mathrm{pmol})$ one day after nerve injury reversed allodynia for more than 24 hours in the early phase, however, no effect on allodynia when given 10 days after nerve injury in the spinal cord [2].MMP-2 maintain NP, implicated in IL-1 $\beta$ cleavage and MMP-2 expression in GFAP+ astrocytes in DRG and spinal cord. Intrathecal administration of TIMP2, an inhibitor of MMP-2, at a very low dose (4.5 pmol), reversed SNL-induced allodynia on day 10 [2].TIMP-1 and TIMP-2 (MMP inhibitors) are powerful therapeutic tools for suppressing NP.

In another study, administration of TIMP1 and TIMP2 has reported to balance enzyme activity of MMP 9 and MMP2, respectively in rat dorsal root ganglia (DRG) following peripheral nerve injury [52]. In situ hybridization exhibited TIMP1 mRNA only localized in DRG satellite cells under normal conditions; however, TIMP1 mRNA increased in satellite cells, and induced within sensory neurons after SNT. Neuronal profiles analysis revealed induced TIMP1 mRNA mainly in small and medium DRG neurons. Induced TIMP1 mRNA predominantly present in activating transcription factor 3 (ATF3)-positive injured DRG neurons. Furthermore, TIMP2 mRNA remained limited within sensory neurons quantitatively lessened at the late phase of PNI.

\section{SIRNA THERAPY}

CED is a method for direct infusion of therapeutics not able to cross the blood-CNS barrier and may be a method for delivering macromolecules such as siRNA [11]. Use of a syringe pump system may allow for low-flow infusions. To ensure success, pressure monitoring may be required. If CED to be MRI guided then the supporting systems for CED such as infusion pumps and pressure monitors would need to be MRI compatible.

The utility of using anionic, siRNA nanoparticles developed from PEGylated liposomes, contemplated of therapeutic significance for treating neuronal diseases as compared to cationic Nano complexes use [53].They evaluated administration of siRNA to BACE1 in the rat brain showed both the silencing of BACE1 and substrate-enzyme specificity and was achieved in vivo following a single CED injection of anionic nanoparticles.

In support of the results of MMP-9 inhibitors, small interfering RNA targeted against MMP-9 in vivo successfully suppressed DRG MMP-9 activity and expression following SNL and delayed the development of allodynia [2]. Conversely, a single injection of exogenous MMP-9 produced rapid mechanical allodynia, which recovered at 6 days. In addition, MMP-9 injections evoked marked microglial activation, as indicated by increased expression of the microglial marker OX-42. MMP-2 treated microglia also showed phosphorylated p38, reversed by subsequent treatment of MMP-9 siRNA treatment. Furthermore, a p38 inhibitor reversed allodynia after MMP-9. Thus, p38 activation in spinal microglia may occur downstream of MMP-9 for mediating nerve injury-induced allodynia. To exclude a role of MMP-9 in late phase NP, researchers used MMP-2 inhibitors in MMP-9 null mice, shown 
to produce more profound anti-allodynia effect in knockout mice, due to MMP-2 compensation in these mice. SNL-induced allodynia reversed by specific MMP-2 knockdown with a siRNA. Finally, ELISA analysis has shown that IL-1 $\beta$ increases following MMP-2 treatment. Conversely, IL-1 $\beta$ decreases after either MMP9 siRNA treatment, p38 inhibitor treatment, or by inhibiting IL- $1 \beta$ signaling with a neutralizing antibody. Collectively, these data suggest that allodynia delayed by administration of MMP-9 inhibitors, such as siRNA, p38 inhibitors, and IL- $1 \beta$ blockers.

The development of dendrimer-conjugated magneto fluorescentNano worms, popularly known as "dendriworms", became a modular platform for testing siRNA delivered to the mouse brain in vivo [54]. CED of siRNA to oligodendrocytes silence an endogenous oligodendrocyte-specific gene; 2', 3'-cyclic nucleotide 3'-phosphodiesterase (CNPase); and increase the flow rate of siRNA infusion enhanced mRNA suppression in white matter regions distant from the infusion site [55]. This study demonstrates the potential of siRNAs in the treatment of CNS disorders, specifically oligodendrocyte pathology. While comparing the dendriworms vs mouse brains receiving the siRNA delivery, epidermal growth factor receptor (EGFR; ErbB-1; HER1 in humans) expression levels confirmed that dendriworms act as a multimodal platform to allow fluorescent tracking of siRNA delivery in vivo.

A trinucleotide (cytosine-adenine-guanine) repeated expansion in the hunting tin (Htt) gene is cause of Huntington's disease [56]. A single administration into the adult striatum of an siRNA targeting $\mathrm{Htt}$ can silence mutant $\mathrm{Htt}$, attenuate neuronal pathology, and delay the abnormal behavioral phenotype observed in a rapid-onset, viral transgenic mouse model. In a non-human primate brain model, the silencing Htt obtained after sufficient distribution of siRNA that target $\mathrm{Htt}$ and high levels of $\mathrm{Htt}$ suppression and, thus, could lead to therapeutic benefit [57].

In glioblastoma cancer research, targeting HIF$1 \alpha$ by siRNA administration in vivo resulted in decreased tumor growth and enhanced survival [58]. The efficacy of IL-13R alpha2 in glioma cells and antitumor activity by cells treated with antisense oligonucleotide or siRNA to IL-13R alpha2 chain considered promising as receptordirected cytotoxic therapy [59]. The data revealed mean tumor size in nude mice receiving intraperitoneal or intratumoral IL-13 cytotoxin was significantly smaller in control tumors and still larger compared to IL-13R alpha2-transfected tumors. However, animals receiving IL-13R alpha2 cDNA intracranial via CED established glioma followed by IL-13 R alpha2 cytotoxin administration by the same route mediated regression and prolonged survival as compared to control animals.

\section{MIRNAS THERAPY FOR TREATING NEUROPATHIC PAIN}

Newly emerging studies relating the regulatory role of miRNAs in protein expression and the neuropathic pain associated with peripheral and central sensitization post-injury [60]show promise and further examine the functional role of individual miRNAs. Neuropathic pain decreased with miR23b infusion that occurs as result of the NOX4 gene inactivated, where MiR23b protects GABAergic neurons against ROS/p38/c-JNK apoptotic cell death [61].A mouse model that utilized NOX4 and miR23b revealed both negative and positive impacts on neuropathic pain. Continually, Spinal nerve ligation (SNL) alters expression levels of miR96, miR-182, and miR-183, suggesting that these molecules related to chronic neuropathic pain through translational regulation of painrelevant genes [62]. MiRNAs are involved in the post-transcriptional regulation of hundreds of genes and their relationship to chronic pain accompanied by significant changes in gene expression in various types of cells [63]. Different disease processes, such as peripheral nerve injury, inflammatory diseases, cancer, and spinal cord injury show an alteration in miRNA expression suggesting that novel treatments could developed to respond to this alteration. Specific miRNAs may develop as new molecular targets for pain prevention and relief [64]. Sequencing of miRNA utilized to identify miRNAs in primary sensory neurons related to neuropathic pain [65]. MiRNAs may participate in the regulatory mechanisms of genes associated with the pathophysiology of chronic pain as well as the nociceptive processing following acute noxious stimulation [66].

MiRNA therapy gained popularity following substantial evidence that miRNAs differentially regulate in both dorsal root ganglia (DRG) and the dorsal horn of the spinal cord under diverse pain states. An up regulation of miR-341 in the DRG and down regulation of miR-203, miR- 
181a-1 and miR-541 in the spinal dorsal horn leads to development of neuropathic pain [29]. Continually, a sciatic nerve ligation (SNL) study shows a drastic decrease in miR200b and miR429 in NAcc neurons [67]. Increased DNMT3a proteins strongly expressed in postsynaptic neurons in the NAcc during a neuropathic pain-like state, as indicated by the presence of DNMT3a-immunoreactivity in the NAcc. The co-relation of upregulation of calcium channel, Cav1.2-comprisingL-type (Cav1.2-LTC) in spinal dorsal horn and downregulation of miRNA-103 implicated in the onset of NP in rats [68].MiR-103 simultaneously regulates the expression of three subunits forming Cav1.2-LTC in this assimilated regulation. In this antithetical association, miRNA-103 knockdown in naïve rat results in hypersensitivity to pain and intrathecal administration of miRNA-103 successfully attenuate pain.

MiR-96 participates in the regulation of neuropathic pain through inhibiting the expression of Nav1.3 in the DRG of CCI rats [69]. A model of chronic constriction injury (CCI) in the spinal cord was used to show an increased amount of miRNA-155, which is used to identify neuropathic pain [70]. Inhibiting miRNA-155 reversed suppression of cytokine signaling 1 (SOCS1), which in turn overturned the normal inhibitory effects of miRNA-155 on neuropathic pain. The study showed that miRNA-155 is an important target in preventing neuropathic pain development. SNL-induced mechanical allodynia significantly correlated with the decreased expression of miR-183 in DRG cells [71]. Replacement of miR-183 downregulates SNL-induced increases in Nav1.3 and BDNF expression and attenuates SNL-induced mechanical allodynia [72] have both reported a 10-fold decrease in miR-203 expression in the spinal dorsal horns but not the dorsal root ganglions, hippocampus, or anterior cingulate cortexes of bCCI rats. Rapla protein expression was upregulated in bCCI rat spinal dorsal horns. MiR-203 directly targets the 3'untranslated region of the rapla gene, thereby decreasing rapla protein expression in neuronlike cells. This reveals the possibility of Rap1a in pain. Furthermore, predisposing miRNA expression patterns and inter-individual variations and polymorphisms in miRNAs and/or their binding sites may serve as biomarkers for pain and help to predict individuals at risk of certain types of pain and responsiveness to analgesic drugs.

In vitro validation for predicted target sites in the 3'-UTR of voltage-gated sodium channel Scn11a, alpha $2 /$ delta1 subunit of voltagedependent $\mathrm{Ca}$-channel, and purinergic receptor P2rx ligand-gated ion channel 4 using luciferase reporter assays showed that identified miRNAs significantly modulate gene expression, suggesting that miRNAs may play a direct role in neuropathic pain [73]. The expression and function of miRNA in different animal pain models needs to consider due to the challenge of application and delivery of miRNA in vivo, the potential toxic effects of miRNA and future problems in clinical application [74]. Furthermore, an existing model of miR-134 shows a balanced expression of MOR1 in DRGs in CFA-induced inflammatory pain, which implies that miR-134 may be a potential therapeutic target for the treatment of neuropathic pain including inflammation [75].

A single miRNA can affect expression of multiple proteins in DRG following peripheral nerve injury exploiting selective proteins may result in acute pain therapy [76]. Testing sural nerve injury by mechanical and cold allodynia or tibia injury by mechanical allodynia, the former injuries resulted in increased expression of seven and decreased expression of four miRNAs. Manipulation of these miRNAs may help prevent neuropathic pain following peripheral nerve injury. Recently, several studies have reported differential expression of miRNAs following different experimental injuries in animal models. Among 373 miRNAs analyzed, 63.7 per cent expressed, however, 13.7 per cent exhibited a change in expression following CCI [77]. Relative decline in expression of miRNA125b on day seven, recovery on day 15 following injury, and minimal changes in expression of miR-132 all demonstrate messenger RNA (mRNA) changes followed by the miRNA changes. The significant changes in expression levels of a several miRNAs in the dorsal horn of the spinal cord of CCI rats contributes towards elucidation of the neuropathic pain mechanisms. In spinal nerve ligated rats, upregulation of miR-195 contributed to lip polysaccharide-induced expression of pro-inflammatory cytokines IL$1 \beta$, TNF- $\alpha$, and iNOS in cultured microglia [78].Upregulation of miR-195 resulted in increased mechanical and cold hypersensitivity 
after PNI, whereas miR-195 inhibition reduced mechanical and cold sensitivity. Out of 111 miRNAs, $68 \%$ reported earlier and $32 \%$ related to the development of tumors of the nervous system and neurodegenerative diseases, few miRNAs, including miR-500, miR-221 and miR-21, relate to CCI-induced NP [79]. In another study miR-134, expression level found inversely related to MOR, the main transcript of $\mu$-opioid receptor (MOR) gene expression [75]. Post inflammatory pain observations included the down-regulation of miR-134 and upregulation of MOR1 in the same tissues. This seems to be the first evidence that intrathecal miR-124 treatment can be used to prevent and treat persistent inflammatory and NP.

Differential expression of hippocampal miRNAs in two rat models comprising of chronic pain chronic constriction injury (CCI) injury and complete Freund's adjuvant (CFA) did not show significant differences in miRNA expression [80]. They recommended further research on investigating correlation among miRNAs, messenger RNAs (mRNAs) and proteins. MiR341 is upregulated in the DRG, whereas miR203, miR-181a-1 and miR-541 are downregulated in the SDH under neuropathic pain conditions [29]. The evidence for nerve injury-induced change by noxious stimuli in expression of miRNAs and Kcna2 antisense RNA in the dorsal root ganglion and its implication in neuropathic pain is discussed [81]. Only three of the miRNAs that were expressed abundantly (rno-miR-30d-5p, rnomiR-125b-5p) or moderately (rno-miR-379-5p) were regulated differentially, making them candidates for novel peripheral nervous system determinants of neuropathic pain. Downregulation of miR-7a is causally involved in maintenance of neuropathic pain through regulation of neuronal excitability, and miR-7a replenishment offers a novel therapeutic strategy specific for chronic neuropathic pain [82]. While monitoring higher expression of miR-21 in injured neurons during chronic neuropathic pain state both mechanical allodynia and thermal hyperalgesia attenuated by intrathecal administration of miR-21 inhibitor [82]. Specific ligation of the left fifth lumbar spinal nerve-induced neuropathic pain showed miR-21 expression in the injured DRG neurons, but not neighboring uninjured DRG neurons, was persistently upregulated following the pain development [63, 82]. MiR-21 is specifically upregulated in the injured DRG neurons and causally involved in the late phase of neuropathic pain. In addition, thermal hyperalgesia and mechanical allodynia could attenuate by administration of miR 21 inhibitor. Discovering the specific roles of microRNAs in chronic pain has future implications in the creation of micro-RNA related drugs to alleviate pain $[63,82]$.

MiRNAs cause gene expressions to switch in key processes of the spinal cord (Nieto-Diaz et al., 2014) [30]. In a rat model, global miRNA expression patterns change as SCI progresses due to the alteration of the expression of cells involved in molecular processes such as apoptosis or astrogliosis. Changes in miRNA expression reported in peripheral nerve injury, inflammatory diseases, cancer, spinal cord injury and neuropathic pain have future implications in the creation of mi-RNA related drugs to alleviate pain [63]. Uncontrollable nociceptive stimulation negatively affects functional recovery in spinal cord injured rats by influencing miRNAs and its associated gene targets [83]. By up-regulating miRNA-124 and miRNA-129, it showed that the miRNAs mediate uncontrollable nociceptive stimulation and utilized in the management of SCI-induced neuropathic pain.

Furthermore, the persistent hyperalgesia in GRK2-deficient mice is associated with an increased ratio of M1/M2 type markers in spinal cord microglia/ macrophages, restored by miR124 treatment. They have proposed that intrathecal miR-124 treatment might be a powerful novel treatment for pathological chronic pain with persistent microglia activation. A decreased level of microglia miR124 observed in LysM-GRK2 +/- mice when hyperalgesia became chronic [84]. Administration of miR-124 prevented persistent pain the mice. The ratio of M1/M2 markers restored with miR-124 treatment. Thus, this treatment is potentially very useful in treating chronic pain. In a rat hippocampi following CCI, out of 373 miRNAs analyzed, 63.5 per cent expressed, and 13.7 per cent changed their expressions after testing mechanical and thermal hypersensitivity on the ipsilateral side [77]. The relative expression of miR-125b decreased to 
$0.70 \pm 0.30$ at day 7 and recovered to $1.65 \pm 0.19$ at day 15. Autophagy signaling of miR-195 represents a novel pathway regulating neuroinflammation, offering a new target for therapy of neuropathic pain [78].

In a diabetic neuropathic pain rat model, some of the induced miRNAs in the lumbar spinal dorsal horn potentially regulate inflammation in genes that were involved in pathogenesis of neuropathic pain [85]. In vivo studies have shown that when deregulated, miRNAs can play a role in post-transcriptional modulation of genes that control pain [28]. In animal models, the dorsal root ganglia, the spinal dorsal horn, and the brain are all areas of microRNA modulation. MicroRNAs have the potential to alleviate pain and serve as markers in disorders such as fibromyalgia and complex regional pain syndrome, as shown through the results of several animal studies [28]. Such changes in miRNAs, which are modulators of posttranscriptional gene expression, can help predict gene expression of targets in the nucleus accumbens (NAcc), a region in the basal forebrain rostral to the area of the hypothalamus [86]. Epigenetic modifications and miRNA modulation may play a role in the neuronal responses and plasticity that induces higher levels of neuropathic pain [87].

\section{Practical Considerations For USE OF CED IN SPINAL CORD}

CED is an advanced infusion technique employed in gel infusion in vitromodels, ex vivo models, and in vivo animal models; adopted for gene therapy of diseases with the goal of delivering therapeutic agents into the brain. Our goal should be to translate the CED techniques developed for brain disorders to a large animal SCI model. This research may help treat patients who suffer NP as well as from a variety of other spinal cord pathologies. Penetration of macromolecules across the blood-spinal cord barrier is limited for several therapeutic compounds that could potentially treat spinal cord disorders that result from injury. Short responses of RNAi illustrate the need for better delivery techniques such as CED. In order to gain a better understanding of chronic pain, a contusion spinal cord injury model be used to evaluate the pain molecules that are expressed as part of an injury induced pain response. The main highlights of the topic are innovation and use of improved delivery methods of gene-based therapeutic agents. Optimization of CED in spinal cord will provide us with the useful information to employ in SCI patients suffering from NP. To examine whether CED-delivered siRNA, which target specific MMPs, supports an enhanced anti-hyperalgesia effect during chronic phase of NP, additional research needs to conduct. The results from this study will impart significant efficacy of employing siRNA via $C E D$, a novel method for delivery in the spinal cord. Perusal of literature reveals no study employing CED technique and its evaluation for delivering miRNAs as therapeutic agents. The successful MRI-aided CED of miRNAs in spinal cord will revolutionize therapeutic delivery techniques not intensively studied.

The animal model employing CED technique in the SCI-induced NP needs to develop for using miRNAs/siRNAs as therapeutic agents for nociceptive genes. The animal studies may include ex vivo and in vivo models involving a shift from small animals like rodents to swine and nonhuman primates. This approach will aid in developing novel therapeutic approaches for the treatment of NP. The spinal cord CED approach should include, i) evaluation of tissue damage associated with catheter insertion and infusion of saline and contrast agent, ii) determination of delivered volume within the spinal cord ( $\mathrm{vd} / \mathrm{vi}$ ratio) with larger molecules (siRNA, miRNAs) and virus-sized particles and iii) determination of the effect of positive flow pressure during insertion i.e. infusion pressure. Results obtained from such studies may lead to successful treatment of NP by CED of appropriate therapeutic agents.

While many promising molecular, gene-, and cell-based therapies explored for SCI related NP employing small animal model. However, delivery of therapeutic agents to specific targets within the spinal cord in humans cannot be performed with intrathecal or intravascular techniques due to the anatomy of the spinal cord in small animals and the blood/spinal cord barrier. Addressing this critical gap associated with targeted drug delivery to the spine cannotbe accomplished in small animal models due to disproportional spinal cord surface to volume ratios where simple diffusion of a pharmaceutical or biologic treatment can have a measureable effect but is not translatable to humans. Promising therapies often fail to demonstrate a substantial effect in the human spinal cord where simple diffusion does not 
deliver the therapy into portions of the transverse spinal cord. Additionally, there is a need to target, visualize, and control delivery in humans to specific gray matter spinal cord tracts to generate the desired treatment while avoiding undesired side effects. Thus, development of promising therapies hampered significantly by two gaps: 1) lack of a relevant large animal model and 2) the lack of a platform for targeted delivery of therapeutics. Most SCI and NPre search occurs in small animal models that result in poor clinical translatability of findings as well as practically limit the development of therapy delivery systems that require modeling of the human spinal cordinanatomy, physiology and size. A validated large animal model of spinal cord pathology would overcome this significant limitation. The genetic proximity of the swine to human, and the anatomical, physiological and path physiological similarities, especially spinalcord anisotropy, surface to volume ratios, and nerve tract organization, make swine the ideal model for preclinical studies of SCI and NP. In addition to non-human primate model, we can aim our research using a translational human-sized swine breed, Wisconsin miniature swine (WMS) ${ }^{\mathrm{TM}}$, developed at UW-Madison by one of authors, Dr. Shanmuganayagam and colleagues, to develop and validate a swine SCI and NPmodel. Develop a variable force impactor that can create controlled and reproducible injury to the spinalcord in our human -sized swine. We will determine the molecular and histopathological changes that occur at the site of SCI in the swine and assess its translational relevance to those observed in humans.

Furthermore, we can plan to utilize the abovedeveloped swine model to develop minimal invasive surgery (MIS) platform for MRI guided CED of the rapeutics in to the spinal cord for SCI and NP. CED is a technique to deliver therapeutics in to neural tissue using continuous positive pressure gradients that expand the interstitial space and increase fluid penetrance providing homogenously distributed infusions within longitudinal segments of the SC. CED injection in the spine has the potential to deliver the rapeutic agents to a larger (rostral-caudal) portion of the dorsal gray matter. Our group has extensive experience in guiding and monitoring CED treatments in the brain, which can be leveraged to advance the process of developing innovative the rapies in the spinalcord. We also plan to make the animal model available as a preclinical resource for use by other SCI researchers to advance their research and development.

Another focus can be developing MRI protocols to quantitatively analyze drug delivery in spinal cord of WMS. We can develop MR acquisition methods that utilize an MR tracer in the infusate (gadolinium-based contrast agents) in the spine rather than simply produce MR contrastweighted images. Our previous work in the brain indicates that different gadolinium -based tracers can accurately match the distribution volume of small molecule drugs, larger molecules like siRNA or proteins, and virussized particles. We can remove confounding factors (B0 and RF in homogeneity) that hamper accuracy. Using standard OR-based stereotactic guidance, we will create spinal infusions in the swine spinal cord with standard conventional infusions and CED infusions. We can measure the quantitative dosage map with subsequent MR and correlated necropsy. We expect a significant portion of the conventional infusion technique will not have localized to the gray matter but will diffuse to white matter tracts. CED-based infusion will treat a much greater volume of the gray matter and minimize distribution to white matter tracts. These results will enable refined development of MRI protocols and analytical approaches.

We need to develop a complete MRI-guided minimally invasive surgical technique for guiding the catheters into the WMS spinal cord at multiple locations. Treatments may require multiple injections along a certain length of the spinal cord. While pre-operative MRI used to guide catheter insertion into the spine using a stereotactic OR-based procedure, registering two geometries where such high sub-mm precision desired and pushes us to a real-time MR-based solution. We can combine technologies to manipulate devices entering the spine from a distance to simplify the MR-guided surgical procedure. An MR-compatible device guidance system will simplify the procedure, as it does not require multiple transitions between an OR for catheter insertion and MR suite for dosage monitoring. Our experience in the brains of non-human primates can be used to guide devices into optimal locations that minimize loss of infusate to nearby white matter tracts. 
We can fabricate the minimally invasive injection hardware platform consisting of two key components: 1) A base device that can be secured and stably attached to the lamina of the spine while allowing stereotaxic adjustments to allow for the angled introduction of the injection catheter into the spinal cord. 2) A fused silica composite injection catheter based on a prototype exclusively available to us along with a catheter introducer made from MRI compatible materials. Here we expect consistently delivered the catheter tip to a 0.7 $\mathrm{mm}$ target within the gray matter of the spinal cord.

We need to predict, monitor, and alter CED infusions in real-time to create ellipsoidal treatment zones using a $5 \mathrm{~cm}$ tract along the WMS spinal cord. CED infusions often spheroidal and will require more sophisticated infusion protocols in the spinal cord where infusate will advect along white matter tracts 10 times faster than in gray matter. We will build upon established, FDA-approved predictive brain transport algorithms to predict the infusion volume for a specific catheter location in the spine where MR maps provide segmented volumes of gray and white matter as an initializer. We will use the quantitative MR dosage maps developed as a real-time indicator of the infusate diffusion during CED delivery. We will modify the existing brain transport algorithms to be predictive of future drug distribution in the spine. This way, surgeons will be able to visualize the likely final distribution for each catheter infusion during therapy, allowing them to alter infusion protocols to assure proper distribution.

Validation of WMS spinal cord CED is of utmost significance. We can establish a surgical platform to CED into the spinal cord of WMS in vivo. We can then evaluate tissue and functional damage associated with catheter insertion and infusion of tracking agents and determine the effect of positive flow pressures during CED through infusion pressure monitoring. Establishing CED methodology in the WMS spinal cord can provide the necessary framework to develop MRI protocols and analytical approaches.

The large surface to volume ratio and small axial plane of the spinal cord in small animals makes the distribution of therapies using currently available delivery systems poorly translatable to humans. Further, small animal models often poorly model the complex pathophysiology associated with neurological disease. The use of a more congruent animal model can mitigate these limitations.

Therapies under consideration for SCI treatment include small molecules like folic acid, siRNAs, TIMPS and virally packaged gene therapies targeting NP associated genes, specifically $M M P-2$ and $M M P-9$. We expect targeted CED of therapies post-SCI will have quantifiable molecular and functional effects in swine. Demonstration of practical utility in a swine SCI model will provide rationale to pursue CED for additional spinal cord pathologies.

\section{REFERENCES}

[1] Narita M, Ikegami D, Yamashita A, Ushijima T. [Global understanding of pain]. Nihon shinkei seishin yakurigaku zasshi $=$ Japanese journal of psychopharmacology 2012; 32:6372.

[2] Kawasaki Y, Xu ZZ, Wang X, Park JY, Zhuang ZY, Tan PH, Gao YJ, Roy K, Corfas G, Lo EH, Ji RR. Distinct roles of matrix metalloproteases in the early- and late-phase development of neuropathic pain. Nature medicine 2008; 14:331-336.

[3] Miranpuri GS SD, Schomberg DT, Alrfaei B, King KC, Rynearson B, Wesley VS, Khan N, Obiakor K, Wesley UV, Resnick DK. Matrix Metalloproteinase 2 Signaling in Spinal Cord Injury-Induced Neuropathic Pain. Annals of Neurosciences 2016; 23:1-6

[4] Clark ZJ, Miranpuri GS, Resnick DK (2010) In Vivo application of RNAi to study pain. Annals of neurosciences 2010; 17:142-145.

[5] Sillay K, Schomberg D, Hinchman A, Kumbier L, Ross C, Kubota K, Brodsky E, Miranpuri G. Benchmarking the ERG valve tip and MRI Interventions Smart Flow neurocatheter convection-enhanced delivery system's performance in a gel model of the brain: employing infusion protocols proposed for gene therapy for Parkinson's disease. Journal of neural engineering 2012; 9:026009.

[6] Brady ML, Raghavan R, Singh D, Anand PJ, Fleisher AS, Mata J, Broaddus WC, Olbricht WL. In vivo performance of a microfabricated catheter for intraparenchymal delivery. Journal of neuroscience methods 2014; 229:76-83.

[7] Kunwar S, Chang SM, Prados MD, Berger MS, Sampson JH, Croteau D, Sherman JW, Grahn AY, Shu VS, Dul JL, Husain SR, Joshi BH, Pedain C, Puri RK. Safety of intraparenchymal convection-enhanced delivery of cintredekin 
besudotox in early-phase studies. Neurosurgical focus 2006; 20:E15.

[8] Rogawski MA. Convection-enhanced delivery in the treatment of epilepsy. Journal of the American Society for Experimental NeuroTherapeutics 2009; 6:344-351.

[9] Miranpuri G, Hinchman A, Wang A, Schomberg D, Kubota K, Brady M, Raghavan R, Bruner K, Brodsky E, Block W, Grabow B, Raschke J, Alexander A, Ross C, Simmons H, Sillay K. Convection Enhanced Delivery: A Comparison of infusion characteristics in $e x$ vivo and in vivo non-human primate brain tissue. Annals of neurosciences 2013; 20:108114.

[10] Miranpuri GS, Kumbier L, Hinchman A, Schomberg D, Wang A, Marshall H, Kubota K, Ross C, Sillay K. Gene-based therapy of Parkinson's disease: Translation from animal model to human clinical trial employing convection enhanced delivery. Annals of neurosciences 2012; 19:133-146.

[11] Sillay K, Hinchman A, Kumbier L, Schomberg D, Ross C, Kubota K, Brady M, Brodsky E, Miranpuri G, Raghavan R. Strategies for the delivery of multiple collinear infusion clouds in convection-enhanced delivery in the treatment of Parkinson's disease. Stereotactic and functional neurosurgery 2013; 91:153-161.

[12] Schomberg D, Wang A, Marshall H, Miranpuri G, Sillay K. Ramped-rate vs continuous-rate infusions: An in vitro comparison of convection enhanced delivery protocols. Annals of neurosciences 2013; 20:59-64.

[13] Richardson RM, Kells AP, Rosenbluth KH, Salegio EA, Fiandaca MS, Larson PS, Starr PA, Martin AJ, Lonser RR, Federoff HJ, Forsayeth JR, Bankiewicz KS. Interventional MRI-guided putaminal delivery of AAV2-GDNF for a planned clinical trial in Parkinson's disease. Molecular therapy: the journal of the American Society of Gene Therapy 2011; 19:1048-1057.

[14] Sampson JH, Brady M, Raghavan R, Mehta AI, Friedman AH, Reardon DA, Petry NA, Barboriak DP, Wong TZ, Zalutsky MR, LallyGoss D, Bigner DD Colocalization of gadolinium-diethylene triamine pentaacetic acid with high-molecular-weight molecules after intracerebral convection-enhanced delivery in humans. Neurosurgery 2011; 69:668-676.

[15] Emborg ME, Joers V, Fisher R, Brunner K, Carter V, Ross C, Raghavan R, Brady M, Raschke J, Kubota K, Alexander A. Intraoperative intracerebral MRI-guided navigation for accurate targeting in nonhuman primates. Cell transplantation 2010; 19:15871597.
[16] Emborg ME, Moirano J, Raschke J, Bondarenko V, Zufferey R, Peng S, Ebert AD, Joers V, Roitberg B, Holden JE, Koprich J, Lipton J, Kordower JH, Aebischer P. Response of aged parkinsonian monkeys to in vivo gene transfer of GDNF. Neurobiology of disease 2009; 36:303-311.

[17] Bjorklund A, Kirik D, Rosenblad C, Georgievska B, Lundberg C, Mandel RJ. Towards a neuroprotective gene therapy for Parkinson's disease: use of adenovirus, AAV and lentivirus vectors for gene transfer of GDNF to the nigrostriatal system in the rat Parkinson model. Brain research 2000; 886:8298.

[18] Valles F, Fiandaca MS, Eberling JL, Starr PA, Larson PS, Christine CW, Forsayeth J, Richardson RM, Su X, Aminoff MJ, Bankiewicz KS (2010) Qualitative imaging of adeno-associated virus serotype 2-human aromatic L-amino acid decarboxylase gene therapy in a phase I study for the treatment of Parkinson disease. Neurosurgery 2010; 67:1377-1385

[19] Hadaczek P, Beyer J, Kells A, Narrow W, Bowers W, Federoff HJ, Forsayeth J, Bankiewicz KS. Evaluation of an AAV2-based rapamycin-regulated glial cell line-derived neurotrophic factor (GDNF) expression vector system. PLoS One 2011;6(11):e27728. doi: 10.1371/journal.pone.0027728. Epub 2011 Nov 21.

[20] Herzog, CD, Brown, L, Gammon, D, Kruegel, B, Lin, R, Wilson, A. et al. Expression, bioactivity, and safety 1 year after adenoassociated viral vector type 2-mediated delivery of neurturin to the monkey nigrostriatal system support cere-120 for Parkinson's disease. Neurosurgery 2009; 64: 602-12; discussion 612

[21] Wang TH, Feng ZT, Wei P, Li H, Shi ZJ, Li LY. Effects of pcDNA3-beta-NGF genemodified BMSC on the rat model of Parkinson's disease. Journal of molecular neuroscience 2008; 35:161-169.

[22] Gasmi M, Herzog CD, Brandon EP, Cunningham JJ, Ramirez GA, Ketchum ET, Bartus RT. Striatal delivery of neurturin by CERE-120, an AAV2 vector for the treatment of dopaminergic neuron degeneration in Parkinson's disease. Molecular therapy: the journal of the American Society of Gene Therapy 2007;15:62-68.

[23] Hall WA, Sherr GT. Convection-enhanced delivery: targeted toxin treatment of malignant glioma. Neurosurgical focus 2006;20:E10.

[24] Ratliff JK, Oldfield EH. Convection-enhanced delivery in intact and lesioned peripheral nerve. Journal of neurosurgery 2001; 95:1001-1011. 
[25] Morrison PF, Chen MY, Chadwick RS, Lonser RR, Oldfield EH. Focal delivery during direct infusion to brain: role of flow rate, catheter diameter, and tissue mechanics. The American journal of physiology 1999; 277:R1218-1229.

[26] Bauknight MW, Chakrabarty S, Hwang BY, Malone HR, Joshi S, Bruce JN, Sander Connolly E, Winfree CJ, Cunningham MG, Martin JH, Haque R. Convection enhanced drug delivery of BDNF through a microcannula in a rodent model to strengthen connectivity of a peripheral motor nerve bridge model to bypass spinal cord injury. J Clin Neurosci. 2012;19(4):563-9.

[27] Carthew RW, Sontheimer EJ. Origins and Mechanisms of miRNAs and siRNAs. Cell 2009;136(4):642-55.

[28] Andersen HH, Duroux M, Gazerani P (2014) MicroRNAs as modulators and biomarkers of inflammatory and neuropathic pain conditions. Neurobiology of disease 2014; 71:159-168.

[29] Li H, Shen L, Ma C, Huang Y (2013) Differential expression of miRNAs in the nervous system of a rat model of bilateral sciatic nerve chronic constriction injury. International journal of molecular medicine 2013; 32:219-226.

[30] Nieto-Diaz M, Esteban FJ, Reigada D, MunozGaldeano T, Yunta M, Caballero-Lopez M, Navarro-Ruiz R, Del Aguila A, Maza RM. MicroRNA dysregulation in spinal cord injury: causes, consequences and therapeutics. Frontiers in cellular neuroscience 2014; 8:53.

[31] Sandberg DI, Edgar MA, Souweidane MM. Convection-enhanced delivery into the rat brainstem. Journal of neurosurgery 2002; 96:885-891.

[32] Vogelbaum MA (2007) Convection enhanced delivery for treating brain tumors and selected neurological disorders: symposium review. Journal of neuro-oncology 2007; 83:97-109.

[33] Pomfret R, Miranpuri G, Sillay K. The substitute brain and the potential of the gel model. Annals of neurosciences 2013; 20:118122.

[34] Pomfret R, Sillay K, Miranpuri G. Investigation of the electrical properties of agarose gel: characterization of concentration using nyquist plot phase angle and the implications of a more comprehensive in vitro model of the brain. Annals of neurosciences 2013; 20:99-107.

[35] Emborg ME, Moirano J, Raschke J, Bondarenko V, R. Zufferey R, et al. Response of Aged Parkinsonian Monkeys to In Vivo Gene Transfer of GDNF Neurobiol Dis. Author manuscript; available in PMC 2010 November 22. Published in final edited form as: Neurobiol. Dis. 2009; 36(2): 303-311.
[36] Dass B, Olanow CW, Kordower JH. Gene transfer of trophic factors and stem cell grafting as treatments for Parkinson's disease. Neurology 2006;66(10 Suppl 4):S89-103.

[37] Rosenberg JB, Sondhi D, Rubin DG, Monette S, Chen A, Cram S, De BP, Kaminsky SM, Sevin C, Aubourg P, Crystal RG. Comparative efficacy and safety of multiple routes of direct CNS administration of adeno-associated virus gene transfer vector serotype rh.10 expressing the human arylsulfatase A cDNA to nonhuman primates. Human gene therapy clinical development 2014; 25:164-177.

[38] Tsujiuchi T, Natsume A, Motomura K, Kondo G, Ranjit M, Hachisu R, Sugimura I, Tomita S, Takehara I, Woolley M, Barua NU, Gill SS, Bienemann AS, Yamashita Y, Toyokuni S, Wakabayashi T. Preclinical evaluation of an O(6)-methylguanine-DNA methyltransferasesiRNA/liposome complex administered by convection-enhanced delivery to rat and porcine brains. American journal of translational research 2014; 6:169-178.

[39] Wood JD, Lonser RR, Gogate N, Morrison PF, Oldfield EH. Convective delivery of macromolecules into the naive and traumatized spinal cords of rats. Journal of neurosurgery 1999; 90:115-120.

[40] Lonser RR, Gogate N, Morrison PF, Wood JD, Oldfield EH. Direct convective delivery of macromolecules to the spinal cord. Journal of neurosurgery 1998; 89:616-622.

[41] Lonser RR, Weil RJ, Morrison PF, Governale LS, Oldfield EH. Direct convective delivery of macromolecules to peripheral nerves. Journal of neurosurgery 1998; 89:610-615.

[42] Cunningham J, Oiwa Y, Nagy D, Podsakoff G, Colosi P, Bankiewicz KS. Distribution of AAV-TK following intracranial convectionenhanced delivery into rats. Cell transplantation 2000; 9:585-594.

[43] Ratliff J. K., Oldfield E. H. Convectionenhanced delivery in intact and lesioned peripheral nerve. J. Neurosurg 2001; 95, 10011011. 10.3171/jns.2001.95.6.1001

[44] Sarntinoranont M, Iadarola MJ, Lonser RR, Morrison PF. Direct interstitial infusion of NK1-targeted neurotoxin into the spinal cord: a computational model. American journal of physiology Regulatory, integrative and comparative physiology 2003; 285:R243-254.

[45] Sarntinoranont M, Chen X, Zhao J, Mareci TH.Computational model of interstitial transport in the spinal cord using diffusion tensor imaging. Ann Biomed Eng. 2006;34(8):1304-21. Epub 2006 Jul 11.

[46] Morrey JD, Siddharthan V, Olsen AL, Wang H, Julander JG, Hall JO, Li H, Nordstrom JL, Koenig S, Johnson S, Diamond MS. Defining 
limits of treatment with humanized neutralizing monoclonal antibody for West Nile virus neurological infection in a hamster model. Antimicrobial agents and chemotherapy 2007; 51:2396-2402.

[47] Kim JH, Astary GW, Chen X, Mareci TH, Sarntinoranont M. Voxelized model of interstitial transport in the rat spinal cord following direct infusion into white matter. Journal of biomechanical engineering 2009; 131:071007.

[48] Chen X, Astary GW, Mareci TH, Sarntinoranont M. Determination of macromolecular concentration following direct infusion into hydrogel using contrast-enhanced MRI. Conference proceedings: Annual International Conference of the IEEE Engineering in Medicine and Biology Society IEEE Engineering in Medicine and Biology Society Annual Conference 2007; 2887-2890.

[49] Chen X, Astary GW, Mareci TH, Sarntinoranont $M$. In vivo contrast-enhanced MR imaging of direct infusion into rat peripheral nerves. Annals of biomedical engineering 2011; 39:2823-2834.

[50] Cachon-Gonzalez MB, Wang SZ, McNair R, Bradley J, Lunn D, Ziegler R, Cheng SH, Cox TM. Gene transfer corrects acute GM2 gangliosidosis--potential therapeutic contribution of perivascular enzyme flow. Molecular therapy: the journal of the American Society of Gene Therapy 2012; 20:1489-1500.

[51] Martin Bauknight W, Chakrabarty S, Hwang BY, Malone HR, Joshi S, Bruce JN, Sander Connolly E, Winfree CJ, Cunningham MG, Martin JH, Haque R. Convection enhanced drug delivery of BDNF through a microcannula in a rodent model to strengthen connectivity of a peripheral motor nerve bridge model to bypass spinal cord injury.J Clin Neurosci. 2012;19(4):563-9.

[52] Huang B, Zhao X, Zheng LB, Zhang L, Ni B, Wang YW. Different expression of tissue inhibitor of metalloproteinase family members in rat dorsal root ganglia and their changes after peripheral nerve injury. Neuroscience 2011; 193:21-8.

[53] Tagalakis AD, Lee do HD, Bienemann AS, Zhou H, Munye MM, Saraiva L, McCarthy D, Du Z, Vink CA, Maeshima R, White EA, Gustafsson K, Hart SL. Multifunctional, selfassembling anionic peptide-lipid nanocomplexes for targeted siRNA delivery. Biomaterials 2014; 35:8406-8415.

[54] Agrawal A, Min DH, Singh N, Zhu H, Birjiniuk A, von Maltzahn G, Harris TJ, Xing D, Woolfenden SD, Sharp PA, Charest A,
Bhatia S. Functional delivery of siRNA in mice using dendriworms. ACS Nano 2009; 3(9): 2495-504.

[55] Querbes W, Ge P, Zhang W, Fan Y, Costigan J, Charisse K, Maier M, Nechev L, Manoharan M, Kotelianski V, Sah DW. Direct CNS delivery of siRNA mediates robust silencing in oligodendrocytes.Oligonucleotides2009;(1):2329. doi: 10.1089/oli.2008.0165.

[56] DiFiglia M, Sena-Esteves M, Chase K, Sapp E, Pfister E, Sass M, Yoder J, Reeves P, Pandey RK, Rajeev KG, Manoharan M, Sah DW, Zamore PD, Aronin N.Therapeutic silencing of mutant huntingtin with siRNA attenuates striatal and cortical neuropathology and behavioral deficits. Proc. Natl. Acad. Sci. U S A. 2007;104(43):17204-9.

[57] Stiles DK, Zhang Z, Ge P, Nelson B, Grondin R, Ai Y, Hardy P, Nelson PT, Guzaev AP, Butt MT, Charisse K, Kosovrasti V, Tchangov L, Meys M, Maier M, Nechev L, Manoharan M, Kaemmerer WF, Gwost D, Stewart GR, Gash DM, Sah DW. Widespread suppression of huntingtin with convection-enhanced delivery of siRNA. Experimental neurology 2012; 233:463-471.

[58] Gillespie DL, Aguirre MT, Ravichandran S, Leishman LL, Berrondo C, Gamboa JT, Wang L, King R, Wang X, Tan M, Malamas A, Lu ZR, Jensen RL. RNA interference targeting hypoxia-inducible factor 1alpha via a novel multifunctional surfactant attenuates glioma growth in an intracranial mouse model. Journal of neurosurgery 2015; 122:331-341.

[59] Kawakami K, Kioi M, Liu Q, Kawakami M, Puri RK. Evidence that IL-13R alpha2 chain in human glioma cells is responsible for the antitumor activity mediated by receptordirected cytotoxin therapy. Journal of immunotherapy 2005; 28:193-202.

[60] Kynast KL, Russe OQ, Geisslinger G, Niederberger E. Novel findings in pain processing pathways: implications for miRNAs as future therapeutic targets. Expert review of neurotherapeutics 2013; 13:515-525.

[61] Im YB, Jee MK, Choi JI, Cho HT, Kwon OH, Kang SK. Molecular targeting of NOX4 for neuropathic pain after traumatic injury of the spinal cord. Cell Death \&Disease 2012; 3:e426.

[62] Aldrich BT, Frakes EP, Kasuya J, Hammond DL, Kitamoto T. Changes in expression of sensory organ-specific microRNAs in rat dorsal root ganglia in association with mechanical hypersensitivity induced by spinal nerve ligation. Neuroscience 2009; 164:711-723.

[63] Sakai A, Suzuki H. Emerging roles of microRNAs in chronic pain. Neurochemistry international 2014; 77:58-67. 
[64] Kress M, Huttenhofer A, Landry M, Kuner R, Favereaux A, Greenberg D, Bednarik J, Heppenstall P, Kronenberg F, Malcangio M, Rittner H, Uceyler N, Trajanoski Z, Mouritzen $\mathrm{P}$, Birklein F, Sommer C, Soreq H. microRNAs in nociceptive circuits as predictors of future clinical applications. Frontiers in molecular neuroscience 2013; 6:33.

[65] Bali KK, Hackenberg M, Lubin A, Kuner R, Devor M. Sources of individual variability: miRNAs that predispose to neuropathic pain identified using genome-wide sequencing. Molecular pain 2014; 10:22.

[66] Kusuda R, Cadetti F, Ravanelli MI, Sousa TA, Zanon S, De Lucca FL, Lucas G (2011) Differential expression of microRNAs in mouse pain models. Molecular pain 7:17.

[67] Imai S, Saeki M, Yanase M, Horiuchi H, Abe M, Narita M, Kuzumaki N, Suzuki T, Narita M. Change in microRNAs associated with neuronal adaptive responses in the nucleus accumbens under neuropathic pain. J.Neurosci. 2011; 31:15294-15299.

[68] Favereaux A, Thoumine O, Bouali-Benazzouz R, Roques V, Papon MA, Salam SA, Drutel G, Leger C, Calas A, Nagy F, Landry $M$. Bidirectional integrative regulation of Cav1.2 calcium channel by microRNA miR-103: role in pain. The EMBO journal 2011; 30:38303841.

[69] Chen HP, Zhou W, Kang LM, Yan H, Zhang L, $\mathrm{Xu} \mathrm{BH}$, Cai WH. Intrathecal miR-96 inhibits Nav1.3 expressions and alleviates neuropathic pain in rat following chronic construction injury. Neurochemical research 2014; 39:76-83.

[70] Tan Y, Yang J, Xiang K, Tan Q, Guo Q. Suppression of MicroRNA-155 Attenuates Neuropathic Pain by Regulating SOCS1 Signaling Pathway. Neurochemical research 2015; 40:550-560.

[71] Lin CR, Chen KH, Yang CH, Huang HW, Sheen-Chen SM. Intrathecal miR-183 delivery suppresses mechanical allodynia in mononeuropathic rats. The European journal of neuroscience 2014; 39:1682-1689.

[72] Li H, Huang Y, Ma C, Yu X, Zhang Z, and Shen L. MiR-203 involves in neuropathic pain development and represses Rap1a expression in nerve growth factor differentiated neuronal PC12 cells. The Clinical journal of pain 2015; 31:36-43.

[73] von Schack D, Agostino MJ, Murray BS, Li Y, Reddy PS, Chen J, Choe SE, Strassle BW, Li C, Bates B, Zhang L, Hu H, Kotnis S, Bingham B, Liu W, Whiteside GT, Samad TA, Kennedy JD, Ajit SK. Dynamic changes in the microRNA expression profile reveal multiple regulatory mechanisms in the spinal nerve ligation model of neuropathic pain. PloS one 2011; 6:e17670.

[74] Tan PH, Pao YY, Cheng JK, Hung KC, Liu CC. MicroRNA-based therapy in pain medicine: Current progress and future prospects. Acta Anaesthesiologica Taiwanica: official journal of the Taiwan Society of Anesthesiologists 2013; 51:171-176.

[75] Ni J, Gao Y, Gong S, Guo S, Hisamitsu T, Jiang $X$. Regulation of mu-opioid type 1 receptors by microRNA134 in dorsal root ganglion neurons following peripheral inflammation. European journal of pain 2013; 17:313-323.

[76] Norcini M, Sideris A, Martin Hernandez LA, Zhang J, Blanck TJ, Recio-Pinto E. An approach to identify microRNAs involved in neuropathic pain following a peripheral nerve injury. Frontiers in neuroscience 2014; 8:266.

[77] Arai M, Genda Y, Ishikawa M, Shunsuke T, Okabe T, Sakamoto A. The miRNA and mRNA changes in rat hippocampi after chronic constriction injury. Pain medicine 2013; 14:720-729.

[78] Shi G, Shi J, Liu K, Liu N, Wang Y, Fu Z, Ding J, Jia L, Yuan W. Increased miR-195 aggravates neuropathic pain by inhibiting autophagy following peripheral nerve injury. Glia 2013; 61:504-512.

[79] Genda Y, Arai M, Ishikawa M, Tanaka S, Okabe T, Sakamoto A. microRNA changes in the dorsal horn of the spinal cord of rats with chronic constriction injury: A TaqMan(R) Low Density Array study. International journal of molecular medicine 2013; 31:129-137.

[80] Hori Y, Goto G, Arai-Iwasaki M, Ishikawa M, Sakamoto A. Differential expression of rat hippocampal microRNAs in two rat models of chronic pain. International journal of molecular medicine 2013; 32:1287-1292.

[81] Lutz BM, Bekker A, Tao YX. Noncoding RNAs: new players in chronic pain. Anesthesiology 2014; 121:409-417.

[82] Sakai A, Suzuki H. Nerve injury-induced upregulation of miR-21 in the primary sensory neurons contributes to neuropathic pain in rats. Biochemical and biophysical research communications 2013; 435:176-181.

[83] Strickland ER, Woller SA, Garraway SM, Hook MA, Grau JW, Miranda RC. Regulatory effects of intermittent noxious stimulation on spinal cord injury-sensitive microRNAs and their presumptive targets following spinal cord contusion. Frontiers in neural circuits 2014; $8: 117$.

[84] Willemen HL, Huo XJ, Mao-Ying QL, Zijlstra J, Heijnen CJ, Kavelaars A. MicroRNA-124 as a novel treatment for persistent hyperalgesia. Journal of neuroinflammation 2012; 9:143. 
[85] Gong Q, Lu Z, Huang Q, Ruan L, Chen J, Liang Y, Wang H, Yue Y, Feng S. Altered microRNAs expression profiling in mice with diabetic neuropathic pain. Biochemical and biophysical research communications 2015; 456:615-620.

[86] Carlson NR. Physiology of Behavior 2013. Boston: Pearson.
[87] Ikegami D, Yamashita A, Narita M. Understanding of the psychiatry in palliative care: dysfunction of the rewarding system under the pain state associated with exacerbating pain. Japanese journal of psychopharmacology 2013; 33:199-204.

Citation: Gurwattan S Miranpuri, Karl Sillay, Wendell B. Lake, Daniel K. Resnick. Practical Considerations of Convection Enhanced Delivery for Novel Therapies Treating Spinal Cord Injury Related Neuropathic Pain. ARC Journal of Neuroscience. 2017; 2(3):13-29. doi:dx.doi.org/10.20431/2456057X.0203004.

Copyright: (C) 2017 Authors. This is an open-access article distributed under the terms of the Creative Commons Attribution License, which permits unrestricted use, distribution, and reproduction in any medium, provided the original author and source are credited. 